\title{
Interference Cancellation Using an Array Feed Design for Radio Telescopes
}

\author{
Chad Hansen, Karl F. Warnick, and Brian D. Jeffs \\ Department of Electrical and Computer Engineering \\ Brigham Young University \\ Provo, UT 84602
}

\section{Introduction}

Radio frequency interference (RFI) is a growing problem for radio astronomers. Major sources include tertestrial radar, navigational aids, and satellite downlinks. Approaches to the mitigation of RFI include adaptive filtering, spatial nulling, and time blanking (e.g., [1]). Many of these algorithms require multiple medium to high gain antennas, which increases the expense and complexity of systems used for astronomical observations. We investigate the use of a phased array feed in order to allow for RFI migitation with a single reflector.

Outside of radio astronomy, array feeds are used to compensate for reflector aberrations, improve the efficiency of off-axis beams, achieve shaped antenna patterns, and electronically synthesize multiple scanned beams for rapid sky coverage. Most commonly, array feeds in radio astronomical applications have employed electrically large, waveguide-type elements with minimal or no signal combining between elements [2-4], although several phased array feeds have been developed or are in prototype states [4-5].

\section{Sensitivity Optimization}

Our primary goal in the design of an array feed is to optimize the array for RFI cancellation while also achieving high sensitivity and beam steering capability. The phased array feed should also produce a higher sensitivity as compared to a single standard waveguide feed. Other design goals include gain stability and low beam shape distortion for steered beams.

Because only limited work has been done on the use of array feeds in radio astronomy, we first seek to determine the attainable sensitivity of an array feed with no intereferer present, relative to the baseline sensitivity of a standard waveguide feed. In simulations, we model the feed elements as Hertzian dipoles. The radiation pattern of a Hertzian dipole is very similar to that of more sophisticated small elements, so the qualitative results should apply to other element types. The reflector used in the simulations is similar to the dishes used at the NRAO Very Large Array (VLA), with diameter $25 \mathrm{~m}$, F/D ratio .36 , and surface distortion $.025 \mathrm{~mm}$ (the VLA dishes are shaped reflectors whereas the dish used in the simulations is a paraboloid). A hexagonal grid was chosen for the array configuration. A spacing of 0.6 wavelengths was chosen because it satisfies the requirement to fully sample focal plane fields [5]. For initial testing, a hexagonal array of 7 elements was used. The reference waveguide feed is a circular, non-ribbed waveguide with a diameter of $1.3 \lambda$, corresponding to a maximum sensitivity for a $25 \mathrm{~m}$ paraboloid. Far fields and focal plane fields are computed using a commercial PTDbased package (TICRA) 
A line-search optimization algorithm was used to maximize sensitivity. Figure 1 shows the maximum sensitivity of the 7-element array and reflector as a function of offset distance relative to the focal plane. Best sensitivity is obtained at the focal plane, although we note that only a small offset is required before the array is smaller than the geometrical optics cone of the fields focused by the reflector. The array feed also attains a higher sensitivity than the waveguide feed. As with the waveguide feed, optimizing sensitivity entails sacrificing gain in order to achieve higher spillover efficiency. In Fig. 1 the gain of the array is $2 \mathrm{~dB}$ below that achieved using the conjugate field match (CFM) method, but the array has higher spillover efficiency and thus higher sensitivity.

For beam seanning applications, a key question is how far a single beam can be steered while still maintaining high sensitivity. This result can be seen in Fig. 2, for arrays at several offset distances relative to the focal plane. The array in the focal plane produces the highest sensitivity, even for the steered beams. Since the beams are all steered electronically, it is possible to form multiple beams simultaneously, which is ideal for rapid sky surveys.

\section{RFI mitigation}

The next step was to use the array to mitigate radio interference. We define effective sensitivity in the presence of RFI as

$$
S_{\text {eff }}(1 / / y)=\frac{G(K / J y)}{T_{s y s}+T_{l m t}}
$$

which differs from sensitivity as usually defined in that interference power is included in the noise temperature. For our simulations, we neglected atmospheric noise and cosmic background noise since they are small compared to the other parameters. We also assumed a receiver temperature of $15 \mathrm{~K}$, where a somewhat low value was chosen to emphasizes the importance of spillover efficiency and RFI mitigation in the effective sensitivity. For the interference, a single Russian global navigation satellite (GLONASS) operating at $1612 \mathrm{MHz}$ was used. The spillover noise was assumed to arrive at the feed from a warm $300 \mathrm{~K}$ background over the solid angle extending from the reflector rim up to the focal plane.

Using spatial filtering algorithms, beamformer array weights were designed to steer the main beam to the boresight direction and suppress the RFI signal. We considered both linear constrained minimum variance (LCMV) and maximum SNR algorithms, although for the above assumptions the two algorithms yield the same beamformer solution. Figure 3 shows the final pattern for the center beam with the interferer at $30^{\circ}$ with an interference-to-noise ratio (INR) of $0 \mathrm{~dB}$ at the center feed element. Figure 4 shows a detail of the null placed in the direction of the interferer. In order to place the null some gain and spillover efficiency is lost. The overall sensitivity drops by $0.8 \mathrm{~dB}$ from the maximum possible sensitivity. The effective sensitivity is stable in the limits of large and small interferer powers, as shown in Fig. 5.

Although the algorithms are able to suppress RFI, some beam shape distortion occurs, as shown in Fig. 6. More advanced algorithms with shape tolerance constraints are required to eliminate this problem. More significantly, the effective sensitivity is not robust relative to interferer angle of arrival. Figure 7 shows that at some angles high effective sensitivity cannot be achieved. In these cases, the center element has a relatively large 
response in the direction of the interferer, so that the outer elements must be weighted larger in order to combine the element signals in such a way that the interference is cancelled. This generally decreases the overall antenna gain significantly. Figure 8 demonstrates this phenomenon. It may be possible to eliminate this drop in effective sensitivity if a larger array were used. It is important to note that these results are general with respect to the choice of beamformer algorithm in that for the model considered LCMV and maximum SNR both lead to optimal sensitivity. Thus, the array itself must be modified in order to improve the sensitivity results.

\section{Conclusions}

The simulations reported in this paper demonstrate that higher sensitivity relative to standard feeds can in principle be achieved using a phased array feed, and that multiple high sensitivity beams can be formed. Using spatial filtering it is also possible to cancel RFI, but beam distortions and sensitivity reduction can occur. Future research is needed to overcome these problems and to study larger arrays, the effects of mutual coupling, and broadband array elements.

\section{References}

[1] C. Barnbaum and R. Bradley, "A New Approach to Interference Excision in Radio Astronomy: Real-time Adaptive Cancellation", Astronomical Journal, November 1998, vol. 116, pp. 2598-2614.

[2] S. J. Blank and W. A. Imbriale, "Array feed synthesis for correction of reflector distortion and vernier beamsteering," IEEE Transactions on Antennas and Propagation, vol. AP-36, pp. 1351-1358, Oct. 1988 .

[3] J. Fisher, "Phased array feeds for low noise reflector antennas," NRAO Electronics Division Internal Report, no. 307, Sept. 1996.

[4] D. Emerson and J. Payne, Eds., Astronomical Society of the Pacific Conference Series, Multi-Feed Systems for Radio Telescopes. San Francisco: Astronomical Society of the Pacific, 1995, vol. 75.

[5] J. Fisher and R. Bradley, "Full sampling array feeds for radio telescopes," Proceedings of the SPIE, Radio Telescopes, vol. 4015, pp. 308-318, 2000.

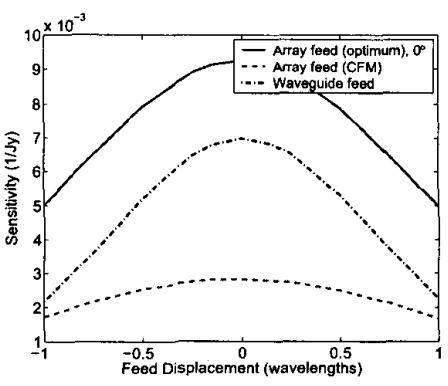

Figure 1: Sensitivity vs. feed displacement from the focal plane. The array feed consists of a 7 element dipole hex array.

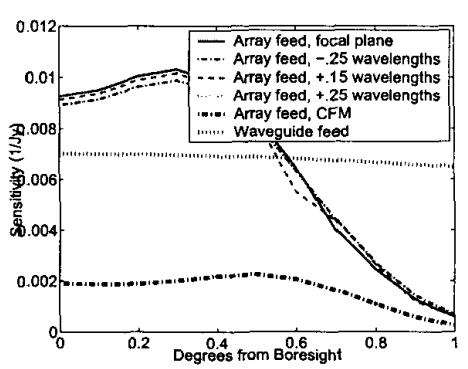

Figure 2: Sensitivity as a function of steered beam angle. 


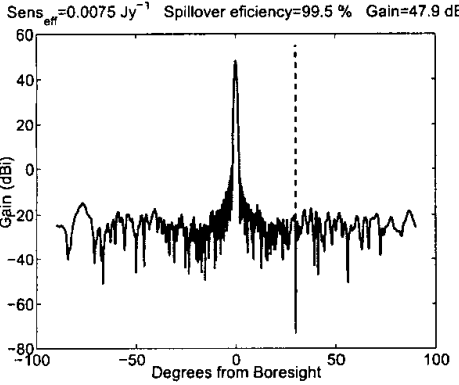

Figure 3: Beam pattern using LCMV to cancel interferer at $30^{\circ}$.

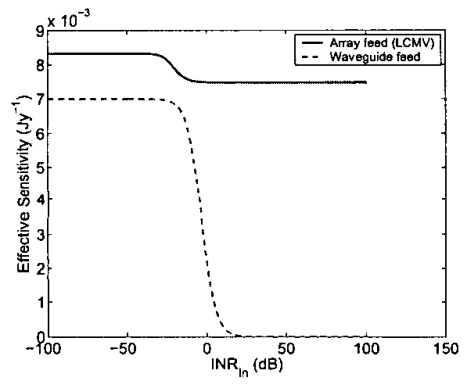

Figure 5: Effective sensitivity vs. interference-to-noise ratio (INR) at the center feed element.

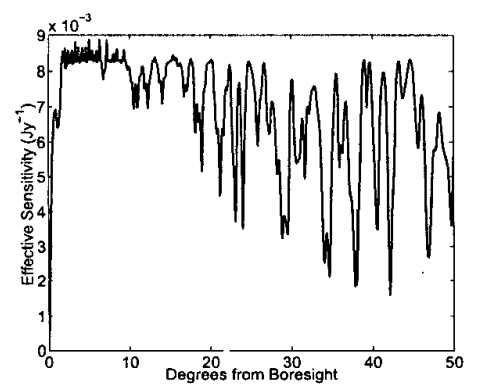

Figure 7: Effective sensitivity as a function of interferer location.

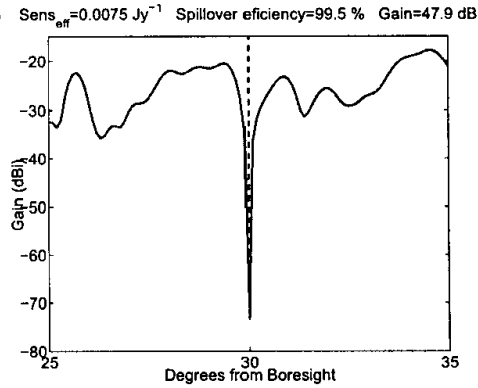

Figure 4: Detail of null in the direction of the interferer.

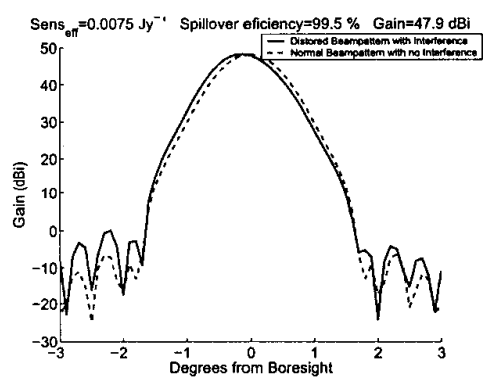

Figure 6: Distorted beam pattern using LCMV. Maximum gain is shifted slightly from $0^{\circ}$.

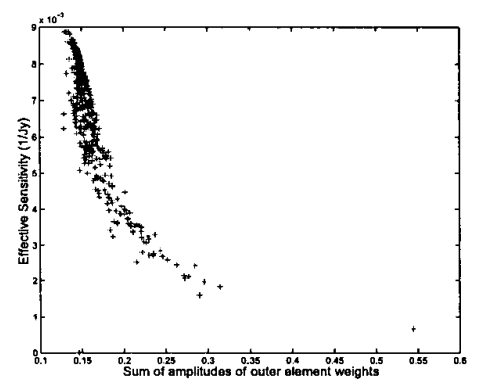

Figure 8: Effective sensitivity vs. amplitudes of outer element weights. When interferer power at the center element is large, outer element weights become large and sensitivity suffers. 